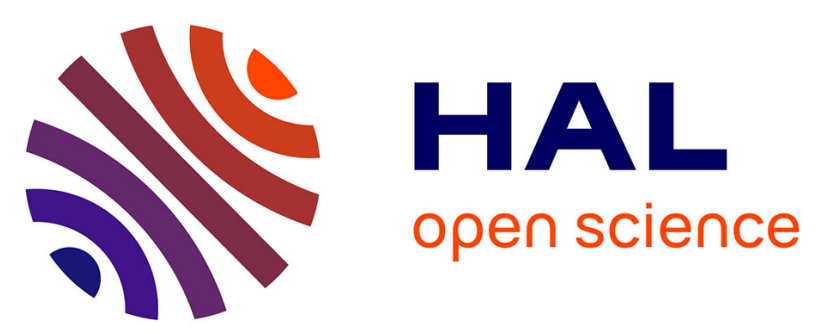

\title{
Connection between singular arcs in optimal control using bridges. Physical occurence and Mathematical model
}

Toufik Bakir, Bernard Bonnard, Jérémy Rouot

\section{- To cite this version:}

Toufik Bakir, Bernard Bonnard, Jérémy Rouot. Connection between singular arcs in optimal control using bridges. Physical occurence and Mathematical model. CDC 2019 - 58th Conference on Decision and Control, Dec 2019, Nice, France. 10.1109/CDC40024.2019.9029880 . hal-02050014v3

\section{HAL Id: hal-02050014 \\ https://hal.inria.fr/hal-02050014v3}

Submitted on 4 Nov 2019

HAL is a multi-disciplinary open access archive for the deposit and dissemination of scientific research documents, whether they are published or not. The documents may come from teaching and research institutions in France or abroad, or from public or private research centers.
L'archive ouverte pluridisciplinaire HAL, est destinée au dépôt et à la diffusion de documents scientifiques de niveau recherche, publiés ou non, émanant des établissements d'enseignement et de recherche français ou étrangers, des laboratoires publics ou privés. 


\title{
Connection between singular arcs in optimal control using bridges. Physical occurence and Mathematical model
}

\author{
T. Bakir and B.Bonnard and J.Rouot
}

\begin{abstract}
In the time minimal control problem, singular arcs are omnipresent to determine the optimal solutions and this leads to the well known turnpike phenomenon [1]. Very recently, this connection between singular arcs using a bang arc was shown to be relevant to saturate a single spin in Magnetic Resonance Imaging. Based on this example we propose a mathematical model to analyze such connection, which is called a bridge. This is applied to compute bridges in the optimization of chemical reaction networks using temperature control.
\end{abstract}

Keywords: Pontryagin maximum principle · Singularity theory · Geometric control

\section{INTRODUCTION}

In this article we consider a real analytic single input control system in $\mathbb{R}^{n-1}$ of the form: $\frac{\mathrm{d} c}{\mathrm{~d} t}=f(c, T), c \in$ $\mathbb{R}^{n-1}, T \in \mathbb{R}$ which is extended using the Goh transformation $u=\frac{\mathrm{d} T}{\mathrm{~d} t}$ onto the control affine system

$$
\frac{\mathrm{d} x}{\mathrm{~d} t}=F(x)+u G(x), x=(c, T) \in \mathbb{R}^{n}
$$

and the set $\mathcal{U}$ of admissible controls is the set of bounded measurable mappings defined on $\left[0, t_{f}(u)\right]$, and valued into $[-1,1]$ (such model being motivated by the control of chemical reaction networks where $c$ is the vector representing the concentrations of the chemical species and $T$ is the temperature).

We consider the time-minimal control problem where $x(0)=x_{0}$ is fixed and $x\left(t_{f}\right) \in N$ where $N$ is the (analytic) terminal manifold. According to the Maximum Principle [11] an optimal solution has to be found among the extremal triplets $(x(\cdot), p(\cdot), u(\cdot)), p \in \mathbb{R}^{n} \backslash 0$ solutions for a.e. $t \in\left[0, t_{f}\right]$ of the pseudo-Hamiltonian system:

$$
\begin{aligned}
& \dot{x}(t)=\frac{\partial H}{\partial p}(x(t), p(t), u(t)), \dot{p}(t)=-\frac{\partial H}{\partial x}(x(t), p(t), u(t)), \\
& H(x(t), p(t), u(t))=M(x(t), p(t))
\end{aligned}
$$

where $H(x, p, u)=p \cdot(F(x)+u G(x))$ is the pseudo Hamiltonian, $\cdot$ stands for the scalar product and $M(x, p)=$ $\max _{|u| \leq 1} H(x, p, u)$ is the maximized Hamiltonian. Moreover $M(x(t), p(t))$ is a non-negative constant along an extremal solution and one has $p\left(t_{f}\right) \perp T_{x\left(t_{f}\right)} N$ (transversality equation).

Toufik Bakir: Univ. Bourgogne Franche-Comté, Le2i Laboratory UMR 6306, CNRS, Arts et Métiers, Dijon, France toufik.bakirdu-bourgogne.fr

Bernard Bonnard: Inria Sophia Antipolis and Institut de Mathématiques de Bourgogne, 9 avenue Savary, 21078 Dijon, France bernard.bonnardeu-bourgogne. fr

Jérémy Rouot: Institut de Mathématiques de Bourgogne and EPF:Graduate School of Engineering, 2 rue F Sastre, 10430 Rosières-présTroyes, France jeremy.rouotegrenoble-inp.org
An extremal triplet is called

Regular: if $u(t)=\operatorname{sign} p(t) \cdot G(x(t))$ a.e. on $\left[0, t_{f}\right]$.

Singular: if $u(t)$ is defined by the implicit relation

$$
p(t) \cdot G(x(t))=0 \text { a.e. on }\left[0, t_{f}\right] .
$$

Note that a general extremal is a concatenation of such subcases.

In the second case, the singular control $u_{s}(t)$ can be obtained differentiating twice the equation (3) as follows. Introducing the Lie bracket of two vector fields $Z_{1}, Z_{2}$ : $\left[Z_{1}, Z_{2}\right](x)=\frac{\partial Z_{1}}{\partial x}(x) Z_{2}(x)-\frac{\partial Z_{2}}{\partial x}(x) Z_{1}(x)$ one gets:

$$
\begin{aligned}
& p(t) \cdot G(x(t))=p(t) \cdot[G, F](x(t))=0 \\
& p(t) \cdot([[G, F], F]+u(t)[[G, F], G])(x(t))=0
\end{aligned}
$$

From (5) if $p(t) \cdot[[G, F], G](x(t))$ is non zero, the singular control $u_{s}(t)$ can be defined as

$$
u_{s}(t)=-\frac{p(t) \cdot[[G, F], F](x(t))}{p(t) \cdot[[G, F], G](x(t))} .
$$

Moreover from the high order Maximum Principle [7], the so-called Legendre-Clebsch condition:

$$
\frac{\partial}{\partial u} \frac{\mathrm{d}^{2}}{\mathrm{~d} t^{2}} \frac{\partial H}{\partial u}=p(t) \cdot[[G, F], G](x(t)) \geq 0
$$

is a necessary time optimality condition.

Connection between singular arcs and regular arcs are authorized to compute the optimal solutions and such connection occur when meeting the switching surface: $\Sigma$ : $p \cdot G(x)=0$. Such a phenomenon was analyzed in the generic case in the seminal article [8] and it can be used in many case to compute the so-called turnpike optimal solution of the form $\sigma_{ \pm} \sigma_{s} \sigma_{ \pm}$where $\sigma_{ \pm}$is a bang arc and $\sigma_{s}$ is a singular arc (the $\operatorname{arc} \sigma_{1} \sigma_{2}$ represents an $\operatorname{arc} \sigma_{1}$ followed by $\sigma_{2}$ ). The objective of this article is to analyze the connection between singular arcs using a bridge that is a bang arc which is tangent to $\Sigma$ at both extremities, which leads to optimal solutions of the form $\sigma_{ \pm} \sigma_{s} \sigma_{ \pm} \sigma_{s} \sigma_{ \pm} \ldots$ This phenomenon was obtained in many cases in the problem of multisaturation of spin particles see [2] or [10] for a single spin, that is to drive the magnetization vector from the North pole to the center of the Bloch ball, where the system is of the form:

$$
\dot{x}=-\Gamma x-u y, \dot{y}=-\gamma(y-1)+u x,|u| \leq 1
$$

with $0<\gamma \leq 2 \Gamma, \Gamma, \gamma$ being the relaxation parameters.

The main contribution of this article is to present this important physical example to derive a mathematical model in dimension two. In particular it can be used to complete our 
programs of optimizing the yield of batch chemical reactors by controlling the temperature [5], [4].

The organization of this article is the following. In section II, we present a recap of the classification of extremals near the switching surface in the so-called fold case [8] which is the basis of the geometric construction of bridges. In section III, the saturation of the spin case is described in details based on [2]. This leads to construct in section IV a planar mathematical model. Occurrence of bridges for chemical networks are computed in the final section.

\section{CONCEPT AND RECAP ABOUT THE CLASSIFICATION OF EXTREMALS [8]}

\section{A. Notations}

We denote by $\sigma_{+}$(resp. $\sigma_{-}$) a bang arc which constant control +1 (resp. -1 ) and $\sigma_{s}$ is a singular arc. We note $\sigma_{1} \sigma_{2}$ an arc $\sigma_{1}$ followed by $\sigma_{2}$. The surface $\Sigma: p \cdot G(x)=0$ is called the switching surface. If $z(\cdot)=(x(\cdot), p(\cdot))$ is an extremal curve on $\left[0, t_{f}\right]$, we note $\Phi(t)=p(t) \cdot G(x(t))$ the switching function (which codes the switching times). Differentiating twice with respect to time one gets:

$$
\begin{aligned}
& \dot{\Phi}(t)=p(t) \cdot[G, F](x(t)), \\
& \ddot{\Phi}(t)=p(t) \cdot([[G, F], F]](x(t))+u(t)[[G, F], G]](x(t))) .
\end{aligned}
$$

From this calculus we deduce:

Ordinary switching time. $t \in\left[0, t_{f}\right]$ is called an ordinary switching time and $z(t)$ an ordinary switching point if $\Phi(t)=0$ and $\dot{\Phi}(t) \neq 0$. From that we deduce:

Lemma 1. In the ordinary case, near $z(t)$ every extremal solution is of the form $\sigma_{+} \sigma_{-}$if $\dot{\Phi}(t)<0$ and $\sigma_{-} \sigma_{+}$if $\dot{\Phi}(t)>0$.

The Fold case. If $\Phi_{\varepsilon}(t)=\dot{\Phi}_{\varepsilon}(t)=0$ and $\ddot{\Phi}_{\varepsilon}(t)=p(t)$. $([[G, F], F]](x(t))+\varepsilon[[G, F], G]])(x(t)) \neq 0, \varepsilon= \pm 1$, the point $z(t)=(x(t), p(t))$ is called a fold point. If moreover $p \cdot G(x)=p \cdot[G, F](x)$ is regular, we have three cases:

- Case 1. parabolic case: $\ddot{\Phi}_{+}(t) \ddot{\Phi}_{-}(t)>0$.

- Case 2. hyperbolic case: $\ddot{\Phi}_{+}(t)>0$ and $\ddot{\Phi}_{-}(t)<0$.

- Case 3. elliptic case: $\ddot{\Phi}_{+}(t)<0$ and $\ddot{\Phi}_{-}(t)>0$.

Moreover denotes by $u_{s}(t)$ the singular control defined by

$$
\left.\left.p(t) \cdot([[G, F], F]](x(t))+u_{s}(t)[[G, F], G]\right]\right)(x(t))=0
$$

one has

Theorem 1. In a neighborhood of $z(t)$ every extremal solution is of the form:

- Parabolic case: $\sigma_{+} \sigma_{-} \sigma_{+}$or $\sigma_{-} \sigma_{+} \sigma_{-}$

- Hyperbolic case: $\sigma_{ \pm} \sigma_{s} \sigma_{ \pm}$

- Elliptic case: each extremal is bang-bang, i.e. of the form $\sigma_{+} \sigma_{-} \sigma_{+} \sigma_{-} \ldots$ but the number of switches is not uniformly bounded.

The concept of bridge. From the previous analysis, a bridge is a bang arc $\left(\sigma_{+}^{b}\right.$ or $\left.\sigma_{-}^{b}\right)$ connecting two different hyperbolic points so that $\sigma_{ \pm} \sigma_{s} \sigma_{ \pm}^{b} \sigma_{s} \sigma_{ \pm}$is an authorized extremal curve.

\section{THE OCCURRENCE OF (OPTIMAL) BRIDGES} IN MAGNETIC RESONANCE IMAGING [2]

We consider the problem of transferring the system from the North pole $N=(0,1)$ to the center $O=(0,0)$ of the Bloch ball so that the system takes the form

$$
F=-\Gamma x \frac{\partial}{\partial x}+\gamma(1-y) \frac{\partial}{\partial y}, G=-y \frac{\partial}{\partial x}+x \frac{\partial}{\partial y} .
$$

where $\Gamma, \gamma$ are the relaxation parameters and $0<\gamma \leq 2 \Gamma$. Note that $F$ is affine with a single stable node equilibrium at the North pole and integral curves of $G$ are circles.

To make the analysis denoting $\delta=\gamma-\Gamma$, we need the following Lie brackets

$$
\begin{aligned}
& {[G, F]=(-\gamma+\delta y) \frac{\partial}{\partial x}+\delta x \frac{\partial}{\partial y}} \\
& {[[G, F], F]=\left(\gamma(\gamma-2 \Gamma)-\delta^{2} y\right) \frac{\partial}{\partial x}+\delta^{2} x \frac{\partial}{\partial y},} \\
& {[[G, F], G]=2 \delta x \frac{\partial}{\partial x}+(\gamma-\delta y) \frac{\partial}{\partial y} .}
\end{aligned}
$$

Singular arcs. They are located on the set $S$ : $\operatorname{det}(G,[G, F])=0$ which is given by $x(\gamma-2 \delta y)=0$. Hence, they are two lines: the $y$-axis of symmetry and the horizontal line $L: y_{0}=\gamma /(2 \delta)$. These lines intersect the Bloch ball $x^{2}+y^{2}<1$ whenever $2 \Gamma>3 \gamma$ and moreover with $y_{0}<0$.

Along the vertical line, the singular control is zero and along $L$ it is given by: $D^{\prime}(x)+u_{s} D(x)=0$ where $D=\operatorname{det}(G,[[G, F], G])$ and $D^{\prime}=\operatorname{det}(G,[[G, F], F])$. Computing we have $u_{s}=\gamma(2 \Gamma-\gamma) /(2 \delta) x$ and $u_{s} \rightarrow \infty$ when $x \rightarrow 0$.

The strict Legendre-Clebsch condition from [7] is used to distinguish between fast and slow displacement direction. Introducing $D^{\prime \prime}=\operatorname{det}(G, F)$ we have: fast if $D D^{\prime \prime}>0$ and slow if $D D^{\prime \prime}<0$.

Computing we have:

- The horizontal line is fast

- The vertical line is fast if $1>y>y_{0}=\gamma /(2 \delta)$ and slow if $y_{0}=\gamma /(2 \delta)>y>-1$.

We represent in Fig.1 the optimal policy to drive the North pole to the center $O$ computed for parameters such that $0<3 \gamma<2 \Gamma$. By symmetry we represent the policy in the domain $x<0$. Note that the bridge occurs at $S_{2}$ before the saturating point $S_{3}$ where $\left|u_{s}\right|=1$. The optimal policy is $\sigma_{+} \sigma_{s}^{h} \sigma_{+}^{b} \sigma_{s}^{v}$ where $\sigma_{s}^{h}, \sigma_{s}^{v}$ are respectively the horizontal and vertical singular arc, $\sigma_{+}^{b}$ is the bridge. This policy can be compared to the standard inversion sequence $\sigma_{+} \sigma_{s}^{v}$ used in practice.

From the previous analysis one use the following in terms of Lie brackets at $\left(0, y_{0}\right)$ which is the intersection of the singular lines:

$$
p \cdot[[G, F], G]\left(0, y_{0}\right)=0 .
$$

Moreover $p \cdot[[G, F], G]\left(0, y_{0}\right)>0$ for $y>y_{0}$ and $<0$ if $y<y_{0}$ and $p$ can be seen on the picture thanks to the relation $p \cdot G(x)=0$. Conditions on the parameters to have a bridge can be obtained computing Lie brackets up to order 4 at $\left(0, y_{0}\right)$. 


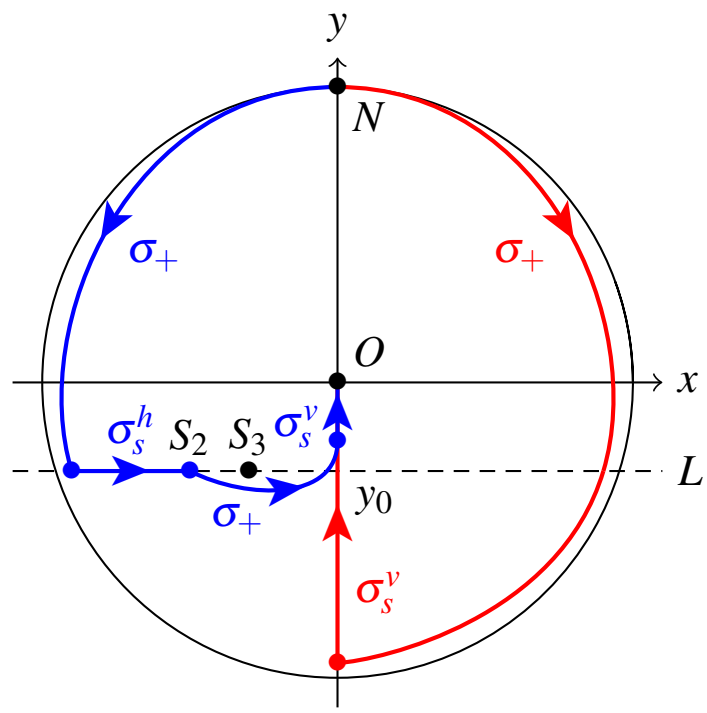

Fig. 1. (left) Time optimal policy with a bridge compared with (right) inversion sequence.

\section{CONSTRUCTION OF A PLANAR (SYMMETRIC) MODEL}

From the previous example we shall construct a local model (near $\left(0, y_{0}\right)$ in the previous example) which exhibits the previous behaviors.

\section{A. Birth of the model.}

We start with the following planar model:

$$
\left\{\begin{array}{l}
\dot{x}=u \\
\dot{y}=1-\varepsilon x^{2}
\end{array}\right.
$$

where the singular arc $\sigma_{s}$ is identified to $t \mapsto(0, t)$ and corresponds to $u_{s}(t)=0$. Such a line is fast if $\varepsilon>0$ and slow if $\varepsilon<0$.

Let us consider now

$$
\left\{\begin{array}{l}
\dot{x}=u \\
\dot{y}=1-x^{2} y .
\end{array}\right.
$$

Computing we have $[G, F]=2 x y \frac{\partial}{\partial y}$ and $[[G, F], G]=$ $2 y \frac{\partial}{\partial y}$. Hence singular trajectories are the two lines: $x=0$ and $y=0$. By construction the vertical line can be followed by $u=0$ and the horizontal line can be followed by $" u=\infty "$ so it cannot be tracked in practice. To construct the model one must bend this line and we get the following.

\section{B. The model}

We consider the system

$$
\dot{\boldsymbol{x}}=F(\boldsymbol{x})+u G(\boldsymbol{x}), t_{f} \leftarrow \min _{|u| \leq 1}, \boldsymbol{x}=(x, y)
$$

where $F=\left(1-x^{2} y\right) \frac{\partial}{\partial y}$ and $G=-(y-1) \frac{\partial}{\partial x}+x \frac{\partial}{\partial y}$. Note that integrating $G$ led to circles centered at $x=0, y=1$.

Computations. We have

- $[G, F](\boldsymbol{x})=\left(-1+x^{2} y\right) \frac{\partial}{\partial x}+\left(2 x y(1-y)+x^{3}\right) \frac{\partial}{\partial y}$,

- $[[G, F], G](\boldsymbol{x})=2 x\left(x^{2}-2(y-1) y\right) \frac{\partial}{\partial x}+\left(x^{2}(5-8 y)+\right.$ $\left.2 y^{3}-4 y^{2}+2 y+1\right) \frac{\partial}{\partial y}$,
- $[[G, F], F](\boldsymbol{x})=\left(x^{2}-x^{4} y\right) \frac{\partial}{\partial x}+x\left(x^{4}+4 x^{2} y^{2}-6 y+\right.$ 2) $\frac{\partial}{\partial y}$,

- $\operatorname{det}(G,[G, F])(\boldsymbol{x})=x\left(x^{2}(1-2 y)+2 y^{3}-4 y^{2}+2 y+1\right)$,

- $D(x, y)=\operatorname{det}(G,[[G, F], G])(\boldsymbol{x})=-2 x^{4}+x^{2}\left(12 y^{2}-\right.$ $17 y+5)-2 y^{4}+6 y^{3}-6 y^{2}+y+1$,

- $D^{\prime}(\boldsymbol{x})=\operatorname{det}(G,[[G, F], F])(\boldsymbol{x})=x\left(x^{4}+x^{2}\left(-4 y^{3}+\right.\right.$ $\left.\left.4 y^{2}-1\right)+6 y^{2}-8 y+2\right)$,

- Collinearity set: $D^{\prime \prime}(\boldsymbol{x})=0$ with $D^{\prime \prime}(\boldsymbol{x})=$ $\operatorname{det}(G, F)(\boldsymbol{x})=(y-1)\left(x^{2} y-1\right)$.

Singular trajectories. The singular set is defined by $\Sigma_{s}=$ $\{q, \operatorname{det}(G,[G, F])(q)=0\}$ and we have

Lemma 2. The singular trajectories corresponds to the union of the vertical line $(O y)$ and the algebraic curve defined by $l(x, y):=x^{2}(1-2 y)+2 y^{3}-4 y^{2}+2 y+1=0$.

Remark 1. The equation $l(x, y)=0$ is equivalent to $x= \pm \sqrt{\frac{2 y^{3}-4 y^{2}+2 y+1}{2 y-1}}$. We denote by $\mathcal{L}$ the horizontal component and by $\mathcal{L}^{\prime}$ the vertical component (see Fig.2).

Moreover $\Sigma_{s} \cap(O y)=\left\{\left(0, y_{0}\right)\right\}$ where $y_{0} \simeq-0.297157$ is the unique real root of $l(0, y)=0$.

Bridge bang arc. Consider the problem of minimizing the time to reach the origin starting from the point $(x(0), y(0))=$ $(-1,-1)$ and under the dynamics defined by (10). A time minimal solution is of the form $\sigma_{-} \sigma_{s} \sigma_{+}^{b} \sigma_{s}$ where $\sigma_{-}$is a bang arc associated to $u=-1, \sigma_{s}$ is a singular arc and $\sigma_{+}^{b}$ is a bridge that is a bang arc connecting two points of the singular line (see Fig.3).

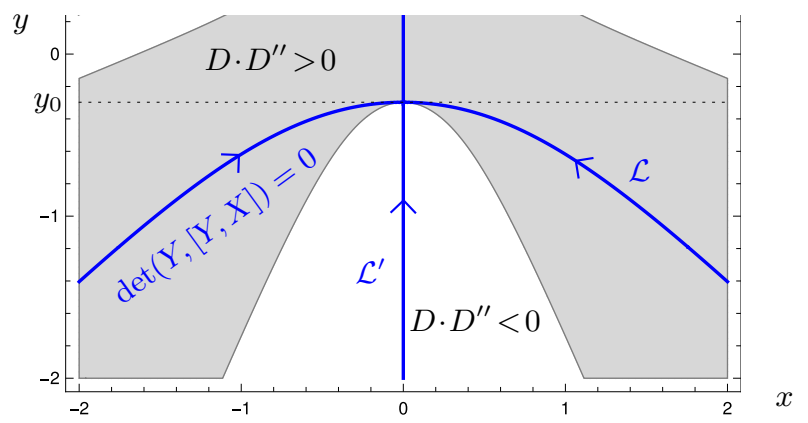

Fig. 2. $(O y)$ and $\mathcal{L}$ are two singular trajectories for the system (10) represented by two continuous lines. The filled regions are the points where $D \cdot D^{\prime \prime}>0$.

From the following computations

- along $(O y), D \cdot D^{\prime \prime}=(y-1)^{2}\left(2 y^{3}-4 y^{2}+2 y+1\right)$,

- along $\mathcal{L}, D \cdot D^{\prime \prime}=2\left(79 y_{0}^{2}-120 y_{0}+74\right)\left(y_{0}-y\right) /(1-$ $\left.2 y_{0}\right)^{4}+o\left(y-y_{0}\right)$

we have

Proposition 1. The vertical singular line $(O y)$ is hyperbolic for $y>y_{0}$, elliptic for $y<y_{0}$. The singular set $\mathcal{L}$ is hyperbolic.

The singular control along $(O y)$ is zero and the expression of the singular control along $\mathcal{L} \cap\{x \leq 0\}$ is

$u_{s}=-\frac{D^{\prime}}{D}=\frac{-8 y^{7}+30 y^{6}-44 y^{5}+38 y^{4}-27 y^{3}+15 y^{2}-6 y+2}{\sqrt{(2 y-1)\left(2 y^{3}-4 y^{2}+2 y+1\right)}\left(8 y^{3}-15 y^{2}+9 y-3\right)}$ 
and there is two saturated points.

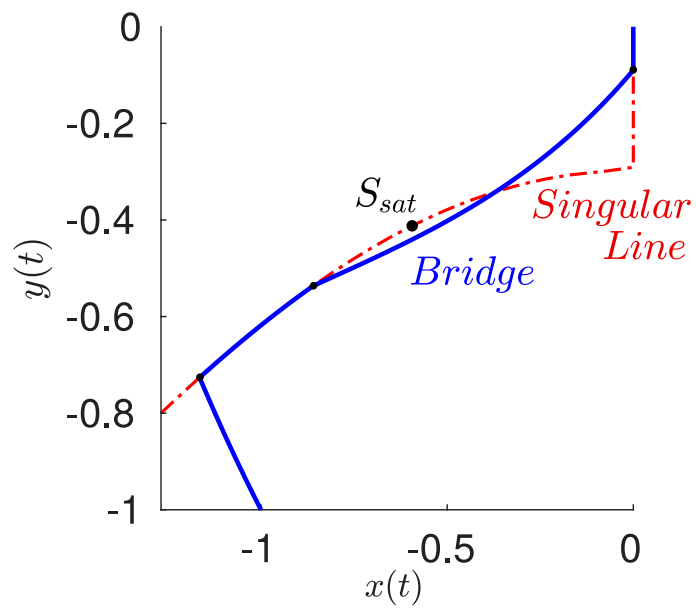

Fig. 3. $\sigma_{+}^{b}$ is a bridge connecting two switching points of the singular set and the singular control saturates at the point $S_{\text {sat }}$.

Remark 2. Adding a parameter $\lambda$ in the model (10) by replacing the vector field $G$ by $G \leftarrow-(y-\lambda) \frac{\partial}{\partial x}+x \frac{\partial}{\partial y}$ affects the position of $y_{0}$ (see Fig.4): as $\lambda \rightarrow+\infty, y_{0} \rightarrow 0$.

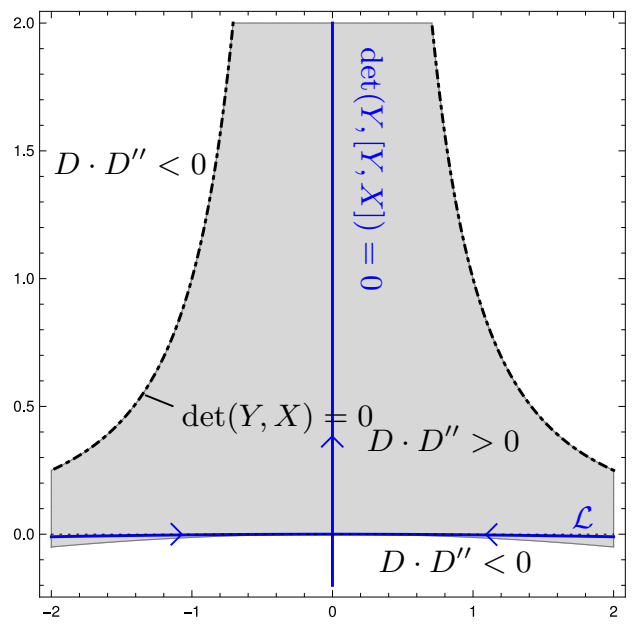

Fig. 4. (Oy) and $\mathcal{L}$ corresponding singular trajectories for the system (10) where $G$ is replaced by $G=-(y-\lambda) \frac{\partial}{\partial x}+x \frac{\partial}{\partial y}$ and $\lambda=200$. The dash-dotted curve corresponds to the collinearity set. The filled regions are the points where $D \cdot D^{\prime \prime}>0$.

By rescaling one can make $y_{0} \rightarrow 0$ and this leads to a complicated synthesis near 0 .

Remark 3. Interaction between two singular lines is coded by the singularities of the flow: $\dot{x}=F(x)-\frac{D^{\prime}(x)}{D(x)} G(x)$ since $D^{\prime}+u_{s} D=0$. It can be analyzed using time reparameterization to lead to $\dot{x}=D(x) F(x)-D^{\prime}(x) G(x)$. This geometric remark is crucial for the extension to the non planar case that we present next.

\section{EXTENSION TO THE $n$-DIMENSIONAL CASE AND AN APPLICATION TO CHEMICAL NETWORKS}

\section{A. Extension}

Due to the space restriction we shall consider the situation $n=3$, which already contains all the technical difficulties. In this case, we introduce the following determinants:

$$
\begin{aligned}
& D=\operatorname{det}(G,[G, F],[[G, F], G]), \\
& D^{\prime}=\operatorname{det}(G,[G, F],[[G, F], F]), \\
& D^{\prime \prime}=\operatorname{det}(G,[G, F], F)
\end{aligned}
$$

and from (4)-(5)-(6) the singular control is given by

$$
u_{s}(x)=-\frac{D^{\prime}(x)}{D(x)} .
$$

We have three types of singular trajectories coming from the fold case classification:

- hyperbolic: $D D^{\prime \prime}>0$

- elliptic: $D D^{\prime \prime}<0$

and the so-called exceptional case corresponds to singular trajectories such that $H=p \cdot F(x)=0$. Eliminating $p$ leads to

- exceptional: $D^{\prime \prime}=0$.

Note that in this case the adjoint vector is either $p$ or $-p$.

Moreover using [3] the small time optimality status is: time minimizing in the hyperbolic and exceptional case, time maximizing in the elliptic case.

Again, since the singular trajectories are solutions of: $\dot{x}=$ $F(x)-\frac{D^{\prime}(x)}{D(x)} G(x)$ connections between singular arcs are related to the behaviors of the solution of the reparameterized equation:

$$
\dot{x}=D(x) F(x)-D^{\prime}(x) G(x)
$$

and in particular near the non isolated equilibria contained in $D=D^{\prime}=0$.

This is the main issue for $n \geq 3$ to analyze connections between singular arcs using a bridge in relation with the occurrence of $p(t) \cdot[[G, F], G](x(t))=0$.

\section{B. Application to chemical networks}

We shall outline the discussion of the occurrence of bridges for chemical networks, extending results from [5], [4]. The problem of maximizing the yield of one species $[X]$ is converted into a minimizing the time to produce a fixed $[X]=d([X]$ denoting the concentration of the species $X)$.

One consider two reactions schemes:

Case 1: $A \stackrel{k_{1}}{\longrightarrow} B \stackrel{k_{2}}{\longrightarrow} C$ : sequence of two irreversible reactions $k_{i}=A_{i} \exp \left(-E_{i} /(R T)\right), i=1,2, A_{i}, E_{i}$ are parameters, $R$ is the gas constant and $T$ is the temperature.

We introduce $v=k_{1}, x=\log [A], y=[B], \alpha=E_{2} / E_{1}$, $\beta=A_{2} / A_{1}^{\alpha}$ so that the dynamics takes the form: $F=$ $-v x \frac{\partial}{\partial x}+\left(v x-\beta v^{\alpha} y\right) \frac{\partial}{\partial y}, G=\frac{\partial}{\partial v}$ and the terminal manifold is $N=\{[B]=y=d\}$. Computing one has

- $[G, F]=x \frac{\partial}{\partial x}+\left(-x+\alpha \beta v^{\alpha-1} y\right) \frac{\partial}{\partial y}$, 


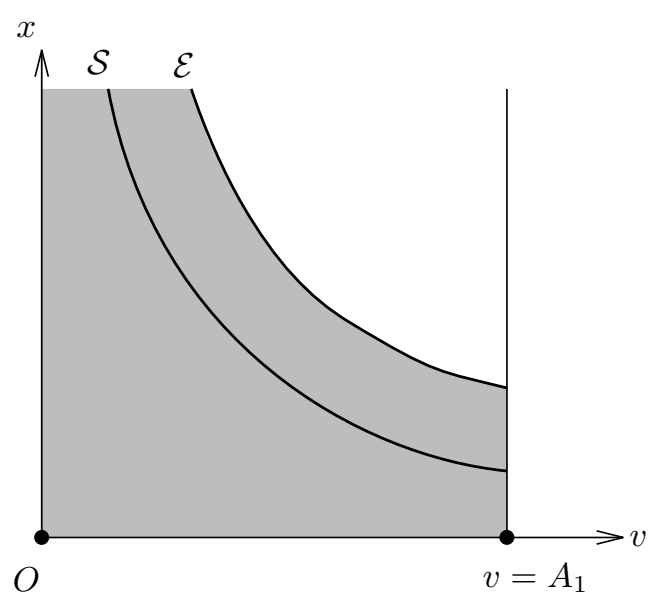

$\alpha<1$

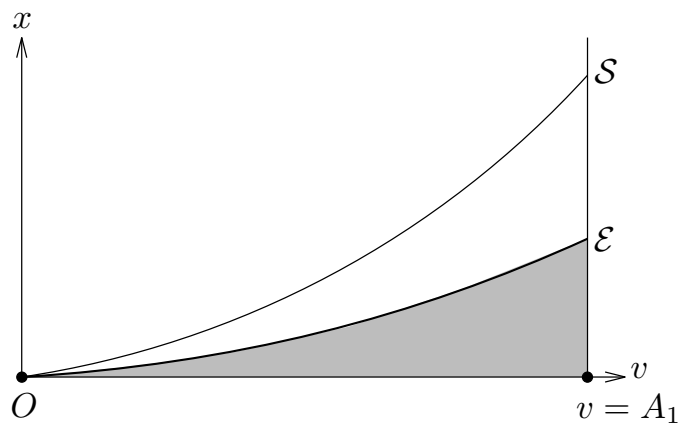

$\alpha>1$.

Fig. 5. Stratification of the manifold $N$.

- $[[G, F], G]=\left(\alpha(\alpha-1) \beta v^{\alpha-2} y \frac{\partial}{\partial y}\right.$,

- $[[G, F], F]=\left((\alpha-1) \beta v^{\alpha} x \frac{\partial}{\partial y}\right.$,

- $D=\operatorname{det}(G,[G, F],[[G, F], G])=\alpha(\alpha-1) \beta x y v^{\alpha-2}$,

- $D^{\prime}=\operatorname{det}(G,[G, F],[[G, F], F])=(\alpha-1) \beta x^{2} v^{\alpha}$,

- $D^{\prime \prime}=\operatorname{det}(G,[G, F], F)=(\alpha-1) \beta x y v^{\alpha}$.

One wants to detect the existence of bridges near the terminal manifold. In the discussion we introduce the following stratification of $N$.

- $\mathcal{E}$ : exceptional set: $n \cdot F=0$ with $n=(0,1,0)$ normal to $N$ and we get $\mathcal{E}: v x-\beta y v^{\alpha}=0$,

- $\mathcal{S}$ : singular arcs so that $n \cdot G=n \cdot[G, F]=0$, that is $-x+\alpha \beta y v^{\alpha-1}=0$.

We represent $\mathcal{E}$ and $\mathcal{S}$ on Fig.5 in the two cases $\alpha>1$ and $\alpha<1$.

Note that $\mathcal{E}$ forms the boundary of the terminal manifold which can be reached from $y(0)<d$. In case $\alpha>1$, there exists no admissible singular arcs and the optimal policy is $u=+1$. In the case $\alpha<1$, there exists hyperbolic singular arcs.

Case 2: Consider now the following network where the first reaction is reversible: $A \underset{k_{3}}{\stackrel{k_{1}}{\longrightarrow}} B \stackrel{k_{2}}{\longrightarrow} C$ where $k_{i}=$ $A_{i} \exp \left(-E_{i} /(R T)\right), i=1,2,3$. Denote $v=k_{1}, \alpha=$ $E_{2} / E_{1}, \alpha^{\prime}=E_{3} / E_{1}, \beta=A_{2} / A_{1}^{\alpha}, \beta^{\prime}=A_{3} / A_{1}^{\alpha}, \mathrm{x}$ $x=[A],[y]=[B]$ so that the dynamics is defined by
$F=\left(-v x+\beta^{\prime} v^{\alpha^{\prime}} y\right) \frac{\partial}{\partial x}+\left(v x-\beta^{\prime} v^{\alpha^{\prime}} y-\beta v^{\alpha} y\right) \frac{\partial}{\partial y}$ and $G=\frac{\partial}{\partial v}$. One has

- $[G, F]=\left(x-\alpha^{\prime} \beta^{\prime} v^{\alpha^{\prime}-1} y\right) \frac{\partial}{\partial x}+\left(-x+\alpha^{\prime} \beta^{\prime} v^{\alpha^{\prime}-1} y+\right.$ $\left.\alpha \beta v^{\alpha-1}\right) \frac{\partial}{\partial y}$,

- $[[G, F], G]=\left(-\alpha^{\prime}\left(\alpha^{\prime}-1\right) \beta^{\prime} v^{\alpha^{\prime}-2} y\right) \frac{\partial}{\partial x}+\left(\alpha^{\prime}\left(\alpha^{\prime}-\right.\right.$ 1) $\left.\beta^{\prime} v^{\alpha^{\prime}-2} y+\alpha(\alpha-1) \beta v^{\alpha-2}\right) \frac{\partial}{\partial y}$,

- $D=\operatorname{det}(G,[G, F],[[G, F], G])=\alpha(\alpha-1) \beta x y v^{\alpha-2}+$ $\alpha^{\prime} \beta^{\prime} \alpha \beta\left(\alpha^{\prime}-\alpha\right) v^{\alpha+\alpha^{\prime}-3} y^{2}$

- $D^{\prime}=\operatorname{det}(G,[G, F],[[G, F], F])=y^{2}\left(\alpha \beta^{2} \beta^{\prime}(\alpha-\right.$ $\left.\alpha^{\prime}\right) v^{2 \alpha+\alpha^{\prime}-2}-\alpha^{\prime} \beta \beta^{\prime 2}\left(\alpha^{\prime}-\alpha\right) v^{\alpha+2 \alpha^{\prime}-2}+\beta\left(\alpha \alpha^{\prime} \beta^{\prime}-\right.$ $\left.\left.\alpha \beta^{\prime}\right) v^{a+\alpha^{\prime}-1}\right)+\beta x y\left(2 \alpha^{\prime} \beta^{\prime}-2 \alpha \beta^{\prime}\right) v^{a+\alpha^{\prime}-1}+(\alpha-$ 1) $\beta x^{2} v^{\alpha}$

- $D^{\prime \prime}=\operatorname{det}(G,[G, F], F)=-\beta v^{\alpha} x y+\left(\alpha^{\prime}-\right.$ a) $\beta \beta^{\prime} v^{\alpha^{\prime}+\alpha-1} y^{2}$.

Computing $\mathcal{E}$ and $\mathcal{S}$ with $y=d$ leads to

- exceptional case: $\mathcal{E}: n \cdot F=v x-v^{\alpha^{\prime}} d\left(\beta v^{\alpha-\alpha^{\prime}}+\beta^{\prime}\right)=$ 0 .

- singular case: $\mathcal{S}: n \cdot[G, F]=d v^{\alpha^{\prime}-1}\left(\alpha \beta v^{\alpha-\alpha^{\prime}}+\right.$ $\left.\alpha^{\prime} \beta^{\prime}\right)-x=0$.

From which we deduce that $\mathcal{E} \cap \mathcal{S}$ is defined by

$$
\begin{aligned}
& x=d \beta^{\prime} v^{\alpha^{\prime}-1} \frac{\alpha-\alpha^{\prime}}{\alpha-1} \\
& v^{\alpha-\alpha^{\prime}}=-\frac{\beta^{\prime}\left(\alpha^{\prime}-1\right)}{\beta(\alpha-1)} .
\end{aligned}
$$

We represent on Fig.6, $\mathcal{E}$ and $\mathcal{S}$ for specific values of the parameters such that

- $\mathcal{E} \cap \mathcal{S} \neq \emptyset$

- A "semi-bridge" occurs and corresponds to a vertical tangent to $\mathcal{S}$ at the point $(x, v)$ where $n$. $[[G, F], G](x, v)=0$.

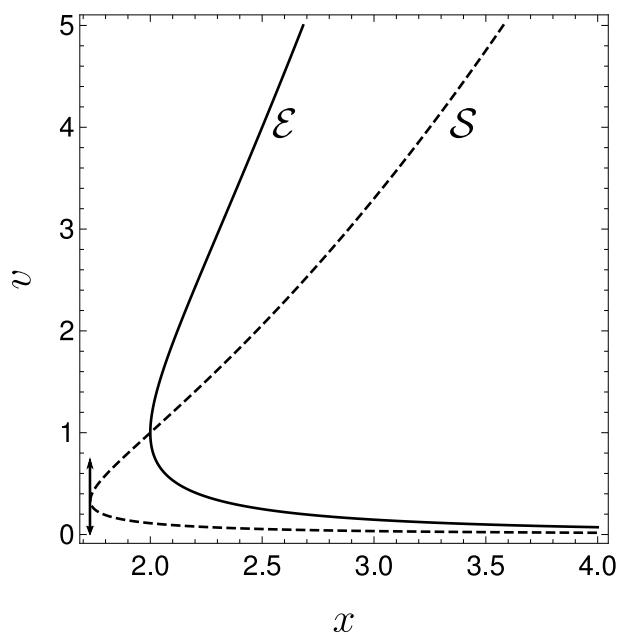

Fig. 6. Stratification of $N: y=d$ for $d=1, \alpha=1.5, \beta=\beta^{\prime}=$ $1, \alpha^{\prime}=0.5 . \mathcal{S}=\{n \cdot G=n \cdot[G, F]=0\}, \mathcal{E}=\{n \cdot F=0\}$.

C. Invariant singular leaf for singular trajectories in the $3 D$ case

Working example for the semi-bridge phenomenon: Next we present a model to analyze the semi-bridge phe- 


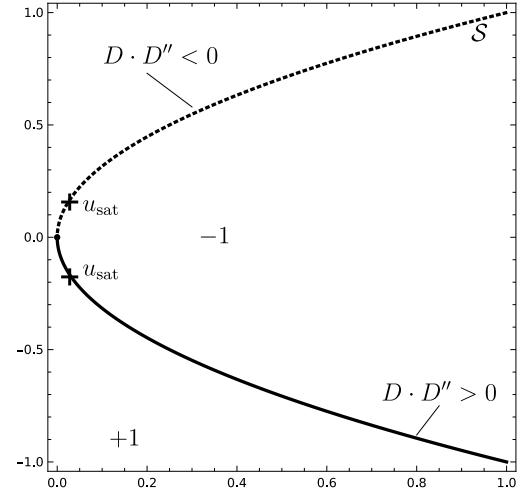

Fig. 7. Stratification of $N$ for the system (11). Dotted line: elliptic, Continuous line: hyperbolic, Crosses: saturating values of the singular control. Parameters values: $a=c=1$.

nomenon

$$
\begin{aligned}
& \dot{x}=1+a y-3 c y z+c z^{3} \\
& \dot{y}=z \\
& \dot{z}=u, \quad|u| \leq 1 .
\end{aligned}
$$

Writing the dynamics as $\dot{q}=F(q)+u G(q), q=(x, y, z)$, we have the following computations

- $F=\left(1+a y-3 c y z+c z^{3}\right) \frac{\partial}{\partial x}+z \frac{\partial}{\partial y}$,

- $G=\frac{\partial}{\partial z}$

- $[G, F]=3 c\left(y-z^{2}\right) \frac{\partial}{\partial x}-\frac{\partial}{\partial y}$,

- $[[G, F], G]=-6 c z \frac{\partial}{\partial x}$,

- $[[G, F], F]=a \frac{\partial}{\partial x}$,

- $D=\operatorname{det}(G,[G, F],[[G, F], G])=-6 c z$,

- $D^{\prime}=\operatorname{det}(G,[G, F],[[G, F], F])=a$.

Proposition 2. The system (11) satisfies the following properties:

- $N: x=0$ is the terminal manifold,

- $G=\frac{\partial}{\partial z}$,

- $\mathcal{S}: n \cdot[G, F](q)=0, x=0$ is $y=z^{2}$,

- $n \cdot[[G, F], G](0)=0$ and $n \cdot[[G, F], F](0) \neq 0$,

- the trajectory associated to $u=0$ defined on $\left[t_{f}, 0\right]$ with $\sigma(0)=0$ is $\gamma \mapsto(t, 0,0)$.

Figure 7 represents the stratification of the terminal manifold for the system (11).

Singular flow: Next we present the main properties of the singular flow.

Proposition 3. The singular control is given by $u_{s}(q)=$ $-D^{\prime}(q) / D(q)=a /(6 c z)$ and the singular trajectories can be integrated by quadratures.

This allows to compute the singular leaf passing through $q(0) \in \mathcal{S}:\left\{y(0)=z(0)^{2}, x(0)=0\right\}$.

We proceed as follows. Parameterizing by $z$, the singular trajectories are solutions of

$$
\begin{aligned}
& \frac{\mathrm{d} y}{\mathrm{~d} z}=6 c z^{2} / a \\
& \frac{\mathrm{d} x}{\mathrm{~d} z}=\left(1+a y-3 c y z+c z^{3}\right) 6 c z / a .
\end{aligned}
$$

From the first equation we deduce, with $y(z(0), z(0))=$ $z(0)^{2}, y(z, z(0))=2 c\left(z^{3}-z(0)^{3}\right) / a+z(0)^{2}$. Plugging such $y$ into the second equation, one gets, with $x(z(0), z(0))=0$, $x(z, z(0))=g(z, z(0))-g(z(0), z(0))$ where $g(z, z(0))=$ $\frac{3 c z^{2}}{5 a^{2}}\left(2 a(2 c+1) z^{3}+30 c z(0)^{2}(a-2 c z(0)) z+30 c^{2} z^{4}+\right.$ $\left.5 a\left(a z(0)^{2}-2 c z(0)^{3}+1\right)\right)$.

\section{CONCLUSION}

In view of applications to optimize the yield of chemical batch reactors we have analyzed the so-called bridge phenomenon in optimal control that is a connection between two singular arcs using a bang arc. Based on the occurrence of this phenomenon in Magnetic Resonance Imaging we have constructed a planar mathematical model to analyze this phenomenon. This is extended in dimension 3 to describe cases where bridges can occur for chemical networks, in relation with reversibility. Note that in this example the singular set is only folded and more complicated chemical schemes have to be used to get ramifications.

\section{REFERENCES}

[1] B. Bonnard, M. Chyba, Singular trajectories and their role in control theory, Mathématiques \& Applications 40 Springer-Verlag, Berlin (2003).

[2] B. Bonnard, O. Cots, J. Rouot, T. Verron, Time minimal saturation of a pair of spins and application in Magnetic Resonance Imaging, Math. Control Relat. Fields (2019), to appear.

[3] B. Bonnard, I. Kupka, Théorie des singularités et optimalité des trajectoires singulières dans le problème temps minimal, Forum Math. 5 (1991) 111-159.

[4] B. Bonnard, G. Launay, Time minimal control of batch reactors, ESAIM: Control, Optimisation and Calculus of Variations 3 (1998) $407-467$.

[5] B. Bonnard, G. Launay, M. Pelletier, Classification générique de synthèses temps minimales avec cible de codimension un et applications, Annales de l'I.H.P. Analyse non linéaire 14 no.1 (1997) 55-102.

[6] U. Boscain \& B. Piccoli, Optimal Syntheses for Control Systems on 2-D Manifolds, Springer SMAI, 43 (2004).

[7] A. J. Krener, The high order maximal principle and its application to singular extremals, SIAM J. Control Optim., 15 no. 2 (1977), 256-293

[8] I. Kupka, Geometric theory of extremals in optimal control problems. I. The fold and Maxwell case, Trans. Amer. Math. Soc. 299, no.1 (1987) 225-243.

[9] I. Kupka, The ubiquity of Fuller's phenomenon, Nonlinear controllability and optimal control, 133 (1990) 313-350.

[10] M. Lapert, Y. Zhang, M. Braun, S. J. Glaser \& D. Sugny, Singular extremals for the time-optimal control of dissipative spin 1/2 particles, Phys. Rev. Lett., 104 no. 2 (2010), 083001

[11] L.S. Pontryagin, V.G. Boltyanskii, R.V. Gamkrelidze, E.F. Mishchenko, The mathematical theory of optimal processes, Oxford, Pergamon Press, 1964.

[12] E.D. Sontag Structure and stability of certain chemical networks and applications to the kinetic proofreading model of T-cell receptor signal transduction IEEE Trans. Automat. Contr. 46 no.7 (2001) 1028-1047.

[13] H.J. Sussmann, Synthesis, presynthesis, sufficient conditions for optimality and subanalytic sets, Nonlinear Controllability and Optimal Control, H.J. Sussmann Ed., Marcel Dekker, New York, 1990 1-20.

[14] J. Zhu, E. Trélat, M. Cerf, Geometric optimal control and applications to aerospace, Pac. J. Math. Ind. 9 no.1 (2017) 8 